\title{
Enumerating Triangulations of Convex Polytopes
}

\author{
Sergei Bespamyatnikh \\ Department of Computer Science, University of British Columbia, 201-2366 Main Mall, Vancouver, B.C., V6T 1Z4, \\ Canada e-mail: bespecs.ubc.ca http://www.cs.ubc.ca/ 〜besp. \\ received January 30, 2001, revised April 10, 2001, accepted April 16, 2001.
}

\begin{abstract}
A triangulation of a finite point set $A$ in $\mathbb{R}^{d}$ is a geometric simplicial complex which covers the convex hull of $A$ and whose vertices are points of $A$. We study the graph of triangulations whose vertices represent the triangulations and whose edges represent geometric bistellar flips. The main result of this paper is that the graph of triangulations in three dimensions is connected when the points of $A$ are in convex position. We introduce a tree of triangulations and present an algorithm for enumerating triangulations in $O(\log \log n)$ time per triangulation.
\end{abstract}

Keywords: polytope, bistellar flip, triangulation, enumeration

\section{Introduction}

Given a finite set of points in $\mathbb{R}^{d}$, a triangulation of $A$ is a geometrically realized simplicial complex which covers the convex hull of $A$ and has their sets of vertices contained in $A$. We are interested in a notion of elementary changes between triangulations of $A$ known as geometric bistellar flips. The bistellar flips arose in the theory of secondary polyhedra developed by Gel'fand, Kapranov and Zelevinsky [GKZ94].

The bistellar flips received considerable attention during the last few years. de Loera et al. [dLSU99] studied how many bistellar flips could a triangulation of configurations with $n$ points in $\mathbb{R}^{d}$ have? In particular, they showed that

1) any triangulation of $n$ points in the plane has at least $n-3$ bistellar flips, and

2) any triangulation of $n$ points in convex position, no three points collinear, has at least $n-4$ bistellar flips.

Santos [San00] constructed sequences of triangulations of point configurations in $\mathbb{R}^{3}$ with $n^{2}+2 n+2$ vertices and only $4 n-3$ geometric bistellar flips.

The question whether one can obtain any triangulation of $A$ from any other by applying a finite sequence of flips is of great importance in areas such as geometric/algebraic combinatorics, computational geometry and optimization, among others. The graph of triangulations of $A$ has the triangulations of $A$ as vertices and the flips between triangulations as edges. Any graph of triangulations in two dimensions is known to be connected since the early days of Computational Geometry [Law77]. Joe [Joe89] considered flips on Delaunay triangulations in three dimensions. The flips of triangulations have applications in mesh 1365-8050 (c) 2001 Maison de l'Informatique et des Mathématiques Discrètes (MIMD), Paris, France 
generation. For a detailed discussion of mesh generation, we refer the reader to the comprehensive survey by Bern and Eppstein [BE92].

de Loera et al. [dLSU99] mentioned a possibility that graph of triangulations can be disconnected in three dimensions even if the points of $A$ are in the vertices of a convex polytope. The main result of this paper is the answer to their question: the graph of triangulations of $n$ points in convex position in $\mathbb{R}^{3}$ is connected. We also present an efficient algorithm for enumerating the triangulations of a convex polytope. The running time is $O(\log \log n)$ per triangulation.

\section{Triangulations and Flips}

Let $A$ be a finite set of points in $\mathbb{R}^{d}$. Without loss of generality we assume that the affine subspace $\operatorname{aff}(A)$ induced by $A$ has dimension $d$.

Definition 1 A triangulation of $A$ is any collection $T$ of affinely independent subsets of $A$ with the following properties:

(i) if $S$ is in $T$, then every subset of $S$ is in $T$, i.e. $T$ is an abstract simplicial complex;

(ii) if $S$ and $S^{\prime}$ are in $T$, then $\operatorname{conv}(S) \cap \operatorname{conv}\left(S^{\prime}\right)$ is a face of both $\operatorname{conv}(S)$ and $\operatorname{conv}\left(S^{\prime}\right)$, i.e. $T$ induces a geometrical simplicial complex in $\mathbb{R}^{d}$;

(iii) $\cup_{S \in T} \operatorname{conv}(S)=\operatorname{conv}(A)$, i.e. $T$ covers the convex hull of $A$.

The subsets of $T$ are called simplices and the simplices of dimension $d$ are maximal simplices. There are four types of simplices in three dimensions, namely, (i) a vertex $S$ if $\operatorname{dim}(S)=0$, (ii) an edge $S$ if $\operatorname{dim}(S)=1$, (iii) a face $S$ if $\operatorname{dim}(S)=2$, and (iv) a simplex $S$ if $\operatorname{dim}(S)=3$. We assume that the points of $A$ are in general position, i.e. there are no three collinear points and there are no four coplanar points. Degenerate cases can be handled by symbolic perturbation [EM90]. The assumption of general position implies that every face in a triangulation $T$ is a triangle and every simplex is a tetrahedron. Recall [Bjö95, (9.9)] the link of a face $F$ in a simplicial complex $T$ is the subcomplex defined by

$$
\operatorname{link}_{T}(F)=\{G \in T, G \cup F \in T, G \cap F=\emptyset\} .
$$

Gel'fand, Kapranov and Zelevinsky [GKZ94] introduced small modifications of triangulations which are called geometric bistellar operations. Following the terminology of matroid theory, we call a minimal affine dependent subset of the in $A$ a circuit, see [BVS ${ }^{+92}$ and [Zie94] for details. Every circuit $Z \subset A$ admits a unique Radon partition $Z=Z_{+} \cup Z_{-}$such that the convex hulls of $Z_{+}$and $Z_{-}$intersect in a point. The pair $\left(Z_{+}, Z_{-}\right)$is called an oriented circuit and, of course, there are only two oriented circuits $\left(Z_{+}, Z_{-}\right)$ and $\left(Z_{-}, Z_{+}\right)$.

The convex hull of a circuit $Z=\left(Z_{+}, Z_{-}\right)$can be triangulated in two ways: $t+(Z)$ as the collection of simplices $\left\{\operatorname{conv}(Z-\{p\}) \mid p \in Z_{+}\right\}$and $t_{-}\left\{\operatorname{conv}(Z-\{p\}) \mid p \in Z_{-}\right\}$.

Definition 2 Let $T$ be a triangulation of $A$ and let $Z=\left(Z_{+}, Z_{-}\right)$be a circuit of $A$. We say that $T$ is supported on $Z$ if the following conditions are satisfied:

(i) One of the triangulations $t_{+}$or $T_{-}(Z)$ is a subcomplex of $T$, and

(ii) All the maximal simplices of $t_{+}(Z)$ (respectively $t_{+}(Z)$ ) have the same link.

If a triangulation $T$ is supported on the circuit $Z$, then we can obtain new triangulation of $A$ as follows: replace the complex $t_{+}(Z) * L$ with the complex $t_{-}(Z) * L$ (and vice versa), where * denotes the operation 


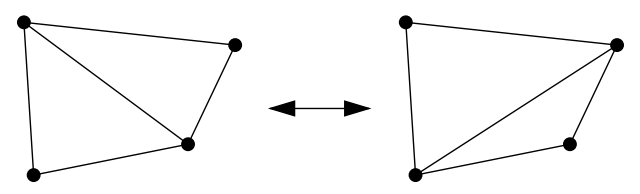

a)

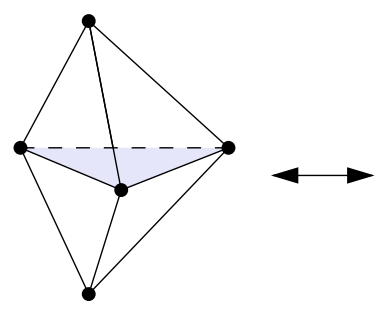

2 tetrahedra

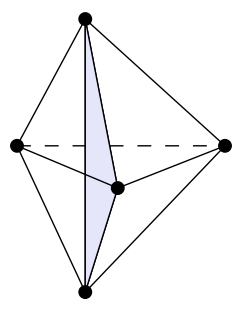

3 tetrahedra

b)

of simplicial join [Bjö95, (9.5)]. The operation of changing the triangulation is called a rigid bistellar flip or geometric bistellar operation.

In this paper we consider the points in convex position in $\mathbb{I}^{3}$. The possible bistellar flips in this case are depicted in Fig 1 b). The flips trade two tetrahedra for three, or vice versa. We will prove that the graph of triangulations is connected. The result is related to the general Baues conjecture [ER98, RZ96] and states that the strict Baues poset for a set $A \subset \mathbb{R}^{3}$ in convex position is connected.

\section{Graph of triangulations}

The main result of this paper is the following.

Theorem 3 Let $P$ be a convex simplicial polytope in $\mathbb{R}^{d}$ and let $T_{1}$ and $T_{2}$ be two triangulations of $P$. The triangulation $T_{2}$ can be obtained from $T_{1}$ using bistellar flips.

We generalize the idea of triangulation tree [HN99, Bes00] which is roughly the following. Let $A$ be a set of $n$ points in general position the plane. We sort the points $p_{1}, p_{2}, \ldots, p_{n}$. Let $T$ be a triangulation of $A$ and let $E$ be the set of edges of $T$. The order of points induces the lexicographical order on $E$. We associate the vector $v(T)=\left(e_{1}, e_{2}, \ldots, e_{m}\right)$ of edges in lexicographical order with the triangulation $T$. Let $T_{\max }$ be the triangulation with lexico-largest vector $v(T)$. If the order of point satisfies the property that $p_{i}, 1 \leq i \leq n$ is a point on the boundary of the convex hull of $\left\{p_{i+1}, p_{i+2}, \ldots, p_{n}\right\}$, then any triangulation $T \neq \bar{T}_{\max }$ admits a flip of type Fig 1 a) increasing the vector of triangulation [Bes00]. The flip making triangulation with lexico-largest vector defines an edge of the triangulation tree. The root of the tree corresponds to the triangulation $T_{\max }$.

Let $A$ be a set of $n$ points in convex and general position in $\mathbb{R}^{3}$. They can be viewed as the vertices of the polytope $P=\operatorname{conv}(A)$ which is convex and simplicial. Let $\left(p_{1}, p_{2}, \ldots, p_{n}\right)$ be any sorted order of points of $A$. We are going to use bistellar flips. Bistellar flips applied to any convex simplicial polytope in the plane have property that the number of simplices of each dimension 0,1 , and 2 is invariant. In dimension $d \geq 3$, however, the number of simplices can be changed when we apply a flip; an example of such bistellar flip is depicted on Fig 1 b). It is not clear that lexicographical order of the triangulations as in the planar case [Bes00] can be used to prove Theorem 3. The key idea now is that we can improve a triangulation using flips and make it closer to a special triangulation (as $T_{\max }$ in the plane). 
To be more specific we introduce some definitions. Let $C$ be any simplicial complex in $\mathbb{R}^{3}$ and $S \subset \mathbb{R}^{3}$. The complex $C$ matches $S$ if, for any simplex of $T$, there is a subsimplex $S^{\prime}$ such that $\operatorname{conv}(S) \cap C=$ $\operatorname{conv}\left(S^{\prime}\right)$ (we may extend the definition to higher dimensions). In other words, the intersection of $C$ and $\operatorname{conv}(A)$ is the union of convex hulls of some simplices of $T$.

Let $P_{i}, 1 \leq i \leq n-3$ be the polytope $\operatorname{conv}\left\{p_{i}, \ldots, p_{n}\right\}$. We assume that the order of points $\left(p_{1}, p_{2}, \ldots, p_{n}\right)$ satisfies the property that $p_{i} \notin P_{i+1}$ for $i=1,2, \ldots, n-4$. A triangulation $T$ has $\operatorname{rank} r=\operatorname{rank}(T)$ if $T$ matches $P_{i}$ for all $i=1,2, \ldots, r$ and $r$ is the maximum number with this property. The notion of rank is well defined and every triangulation $T$ of $A$ has a rank since $T$ matches $P_{1}=\operatorname{conv}(A)$. Clearly, there is unique triangulation $T$ of the rank $n-3$. It can be obtained by the following incremental procedure. We start with the tetrahedron $\left\{p_{n-3}, p_{n-2}, p_{n-1}, p_{n}\right\}$. In general, suppose that we obtained a triangulation of the point set $\left\{p_{i+1}, p_{i+2}, \ldots, p_{n}\right\}$. To obtain triangulation of $\left\{p_{i}, p_{i+1}, \ldots, p_{n}\right\}$ we add the tetrahedron $S=\left\{p_{i}, p_{a}, p_{b}, p_{c}\right\}$ for each boundary triangle $p_{a} p_{b} p_{c}$ of $P_{r+1}$ that is visible from $p_{i}$, and the faces and edges of $S$.

For a triangulation $T$ of rank $r<n-3$, we define the complementary polytope $T_{\text {comp }}$ as the union of the tetrahedra avoiding points $p_{1}, p_{2}, \ldots, p_{r}$, i.e.

$$
T_{\text {comp }}=\cup_{S \in T \cap\left\{p_{r+1}, p_{r+2}, \ldots, p_{n}\right\}} \operatorname{conv}(S) .
$$

The volume of the complementary polytope is the measure that can be used to improve a triangulation.

Theorem 4 Let $A$ be a set of $n$ points in general and convex position in $\mathbb{R}^{3}$ and let $T$ be a triangulation of A. If $T$ has rank less that $n-3$, then there exists a flip which either

(i) increases the rank of $T$, or

(ii) increases the volume of the complementary polytope and preserves the rank of $T$.

We will prove Theorem 4 in the next Section. The connectivity of the graph of triangulations $G(A)$ (Theorem 3) follows from Theorem 4 . Actually we can construct a tree of triangulations whose vertices correspond to the triangulations of $A$ and edges defined as follows. For each triangulation of rank less than $n-3$, we assign a flip increasing either its rank or the volume of its complementary polytope by Theorem 4. We call any flip satisfying these conditions as improving flip. We can assign the vector $\left(\operatorname{rank}(T), \operatorname{vol}\left(T_{\text {comp }}\right)\right)$ to each triangulation of $A$, where $\operatorname{vol}(P)$ is the volume of a polytope $P$. The tree of triangulations can be viewed as the rooted tree with the root corresponding to the triangulation of rank $n-3$. The root triangulation is unique and is denoted as $T_{\text {root }}$.

\section{Existence of an improving flip}

Let $A$ be a set of $n$ points in convex (and general) position in $\mathbb{R}^{3}$ and let $T$ be a triangulation of $A$ with rank $r<n-3$. The complementary polytope $T_{\text {comp }}$ of $T$ differs from the convex hull of the set $\left\{p_{r+1}, \ldots, p_{n}\right\}$, i.e. $T_{\text {comp }} \neq P_{r+1}$. The polytope $T_{\text {comp }}$ contains the vertices of the convex polytope $P_{r+1}$. Since $P_{r+1}$ is unique convex polytope with vertices from $\left\{p_{r+1}, \ldots, p_{n}\right\}$ and containing $\left\{p_{r+1}, \ldots, p_{n}\right\}$, the polytope $T_{\text {comp }}$ is non-convex. We show that any non-convex polytope has at least one non-convex edge. An edge $e$ of a polytope $P \in R^{3}$ is called non-convex if the dihedral angle formed by the planes passing through two faces containing $e$ is greater than $\pi$ (the dihedral wedge contains the neighborhood of $e$ in $P$ ).

Lemma 5 A polytope $P$ in $\mathbb{R}^{3}$ is convex if and only if all its edges are convex. 
Proof: Clearly, each edge of a convex polytope is convex. We show that a non-convex edge of a polytope $P$ implies that $P$ is not convex. Let $\pi$ be the plane passing through a face incident to a non-convex edge of $P$. There are vertices on both sides of $\pi$ and, thus, $P$ is not the intersection of halfspaces bounded by the face planes. Hence $P$ is not a convex polytope.

By definition of rank, the triangulation $T$ matches the polytope $P_{r}=\operatorname{conv}\left(\left\{p_{r}, \ldots, p_{n}\right\}\right)$. Therefore, $T$ matches the polytope $Q$ which is the closure of $P_{r} \backslash T_{\text {comp }}$. The triangulation $T$ matches the polytope $Q$ and induces the triangulation of $Q$.

Let $e$ be any non-convex edge of the polytope $T_{\text {comp. }} e$ is an edge of $Q$ too since $P_{r}$ is convex and $e$ is not an edge of $P_{r}$ by Lemma 5. Let $Z(e)$ be the circuit containing five points: $p_{r}$, endpoints of $e$ and two vertices of the faces (triangles) of $Q$ incident to $e$. The circuit $Z(e)$ is a potential source of improvement of the triangulation $T$ : if $T$ is supported on $Z(e)$ we may apply its flip. There is a simple case when we can apply flip.

The intersection of $Q$ and $T_{\text {comp }}$ is a 2-dimensional manifold. Let $H$ be the boundary of $Q \cap T_{\text {comp }} ; H$ is the horizon of $P_{r+1}$ visible from $p_{r}$. If $e$ has both endpoints in the horizon, then $T$ can be improved by flipping $Z(e)$.

Lemma 6 Let $T$ be a triangulation of $A$ in $\mathbb{R}^{3}$ and $\operatorname{rank}(T)<n-3$. Let e be a non-convex edge of $T_{\text {comp. }}$. If the endpoints of e lies on the boundary of $P_{r}$, then $T$ is supported by the circuit $Z(e)$ and its flip increases the objective $\left(\operatorname{rank}(T), \operatorname{vol}\left(T_{\text {comp }}\right)\right)$.

Proof: Let $p_{a}$ and $p_{b}$ be the endpoints of $e$ and let $p_{c}$ and $p_{d}$ be the remaining vertices of $Z(e)$. Let $B(e)$ be the union of two tetrahedra $p_{r} p_{a} p_{b} p_{c}$ and $p_{r} p_{a} p_{b} p_{d}$. The edge $p_{a} p_{b}$ of $B(e)$ is convex. The edges $p_{r} p_{a}$ and $p_{r} p_{b}$ are convex by their choice. The remaining edges are convex since they are edges of two tetrahedra. By Lemma 5 the biprism $B(e)$ is convex and the segment $p_{c} p_{d}$ lies inside $B(e)$. Hence the segment $p_{c} p_{d}$ intersects the triangle $p_{r} p_{a} p_{b}$ and we can apply flip on $Z(e)$. As a result the tetrahedron $p_{a} p_{b} p_{c} p_{d}$ could be moved from $Q$ to $T_{\text {comp. Lemma follows. }}$

In general, the endpoints of $e$ could not lie on the boundary of $P_{r}$. Consider Radon partition of $Z(e)=$ $\left(Z_{+}, Z_{-}\right)$. We show that neither $Z_{+}$nor $Z_{-}$has exactly one vertex.

Lemma 7 Let $T$ be a triangulation of $A$ in $\mathbb{R}^{3}$ and $\operatorname{rank}(T)<n-3$. Let e be a non-convex edge of $T_{\text {comp }}$ and $T$ is not supported on the circuit $Z(e)=\left(Z_{+}, Z_{-}\right),\left|Z_{+}\right| \leq\left|Z_{-}\right|$. Then

(i) $\left|Z_{+}\right|=2$ and $\left|Z_{-}\right|=3$, and

(ii) $Z_{+}$contains $p_{r}$ and one of the endpoints of $e$.

Proof: Let $p_{a}$ and $p_{b}$ be the endpoints of $e$ and let $p_{c}$ and $p_{d}$ be the remaining vertices of $Z(e)$. 
(i) By the property of Radon partition there are two cases in $\mathbb{R}^{3}$ : either $\left|Z_{+}\right|=1$ and $\left|Z_{-}\right|=4$, or $\left|Z_{+}\right|=2$ and $\left|Z_{-}\right|=3$. Suppose that $\left|Z_{+}\right|=1$. The point $p_{r}$ could not be in $Z_{+}$since it lies on the boundary of the convex polytope $P_{r}$. Suppose that $p_{r} \in Z_{-}$, see Fig. 2. The edge $e$ could not be on the boundary of $\operatorname{conv}(Z(e))$ since the triangle formed by $p_{r}$ and $e$ is common face of tetrahedra $\left(p_{r}, p_{a}, p_{b}, p_{c}\right)$ and $\left(p_{r}, p_{a}, p_{b}, p_{d}\right)$. Thus $e$ lies inside the tetrahedron $\operatorname{conv}(Z(e))$ and is not convex edge of $Q$. Contradiction.

(ii) Let $\Pi$ be the plane passing through $p_{r}, p_{a}$ and $p_{b}$. The points $p_{c}$ and $p_{d}$ lie on different sides of $\Pi$. In other words, the segment $\left[p_{c} p_{d}\right]$ intersects the plane $\Pi$ at point, say $q$. Since $e$ is the convex edge of the polytope $Q$, the points $q$ and $p_{r}$ lies on the same halfplane of $\Pi$ defined by the line passing through $p_{a}$ and $p_{b}$. There are four cases of location of the point $q$.

Case 1. The point $q$ lies in the triangle $\left(p_{r} p_{a} p_{b}\right)$. Then $Z_{+}=\left\{p_{c}, p_{d}\right\}$ and $Z_{-}=\left\{p_{r}, p_{a}, p_{b}\right\}$ and we may apply flip on $Z(e)$. We change two tetrahedra $\Delta_{c}=\operatorname{conv}\left(p_{r}, p_{a}, p_{b}, p_{c}\right)$ and $\Delta_{d}=\operatorname{conv}\left(p_{r}, p_{a}, p_{b}, p_{d}\right)$ by three tetrahedra $\operatorname{conv}\left(p_{r}, p_{c}, p_{d}, p_{a}\right), \operatorname{conv}\left(p_{r}, p_{c}, p_{d}, p_{b}\right)$ and $\operatorname{conv}\left(p_{a}, p_{b}, p_{c}, p_{d}\right)$, see Fig 3 a).

Case 2. $q$ is on right sides of the rays $\left[p_{r} p_{a}\right)$ and $\left[p_{r} p_{b}\right)$. The intersection of the segment $p_{r} p_{b}$ and the triangle $p_{a} p_{c} p_{d}$ is a point and, thus, $Z_{+}=\left\{p_{r}, p_{b}\right\}$ and $Z_{-}=\left\{p_{a}, p_{c}, p_{d}\right\}$, see Fig $3 \mathrm{~b}$ ).

Case 3. $q$ is on left sides of the rays $\left[p_{r} p_{a}\right)$ and $\left[p_{r} p_{b}\right)$. Symmetrically, $Z_{+}=\left\{p_{r}, p_{a}\right\}$ and $Z_{-}=$ $\left\{p_{b}, p_{c}, p_{d}\right\}$, see Fig $\left.3 \mathrm{c}\right)$.

Case 4. $q$ is on left side of the ray $\left[p_{r} p_{a}\right)$ and on the right side of the ray $\left[p_{r} p_{b}\right)$, see Fig $\left.3 \mathrm{~d}\right)$. The point $p_{r}$ lies in the tetrahedron $p_{a} p_{b} p_{c} p_{d}$. Contradiction.

The edges of the polytope $Q$ can be viewed as planar graph $G_{Q}$. The outer face is bounded by edges corresponding the horizon $H$. Let $G_{Q}^{\prime}$ be the subgraph of $G_{Q}$ induced by non-convex edges of $T_{\text {comp. }}$. Lemma 7 allows to orient the edges of $G_{Q}^{\prime}$ as follows. An edge $e=\left(p_{a}, p_{b}\right)$ is directed from $p_{a}$ to $p_{b}$ if $Z_{+}(e)=\left\{p_{r}, p_{b}\right\}$. We call the point $p_{a}$ the initial vertex and the point $p_{b}$ the terminal vertex of $e$.

Lemma 8 Suppose that $T$ is not supported on any circuit $Z(e), e \in G_{Q}^{\prime}$.

(i) For any directed edge $\left(p_{a} p_{b}\right)$ of $G_{Q}^{\prime}$, there exists a directed edge of $G_{Q}^{\prime}$ with initial vertex $p_{b}$.

(ii) A vertex can be terminal for at most one edge of $G_{Q}^{\prime}$.

Proof: (i) Consider edges of $G_{Q}$ incident to $p_{b}$ except for the edge $\left(p_{a} p_{b}\right)$. Let $\left(p_{b} p_{i}\right)$ be the edge making the largest angle to the plane $\Pi\left(p_{b} p_{c} p_{d}\right)$ passing through the points $p_{b}, p_{c}$, and $p_{d}$, see Fig $\left.3 \mathrm{~b}\right)$. The segment $\left(p_{b} p_{i}\right)$ is the convex edge of the polytope $Q$ since both faces of $Q$ incident to $\left(p_{b} p_{i}\right)$ lie on the same side of the plane $\Pi\left(p_{b} p_{c} p_{d}\right)$.

Thus $\left(p_{b} p_{i}\right)$ is an edge of $G_{Q}^{\prime}$. We show that it is oriented so that $p_{b}$ is the initial vertex. Suppose to the contrary that $p_{b}$ is the terminal vertex of $\left(p_{b} p_{i}\right)$. Let $\Pi_{\perp}$ be the plane orthogonal to the line $p_{r} p_{b}$ and passing through the point $p_{b}$. We project each edge of $G_{Q}$ incident to $p_{b}$ onto the plane $\Pi_{\perp}$. Note that the sum of two angles $\angle p_{a} p_{b} p_{d}$ and $\angle p_{a} p_{b} p_{c}$ is greater than $\pi$ in the projection, see Fig. 4 . There are two cases.

Case 1. The point $p_{i}$ is different from $p_{c}$ and $p_{d}$. The sum of two angles formed by $p_{b} p_{i}$ and two adjacent edges on the projection plane is greater than $\pi$ by argument similar to the edge $p_{a} p_{b}$. The four angles formed by $p_{b} p_{i}$ and $p_{a} p_{b}$ are disjoint and their sum is greater than $2 \pi$. Contradiction. 


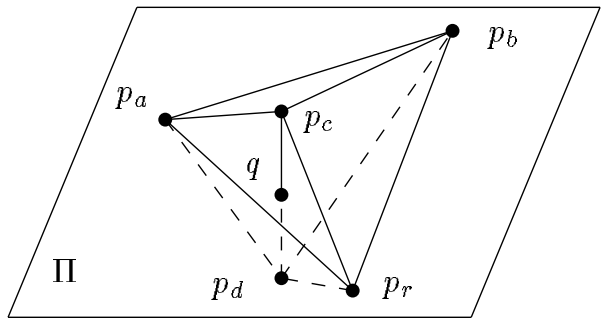

a)

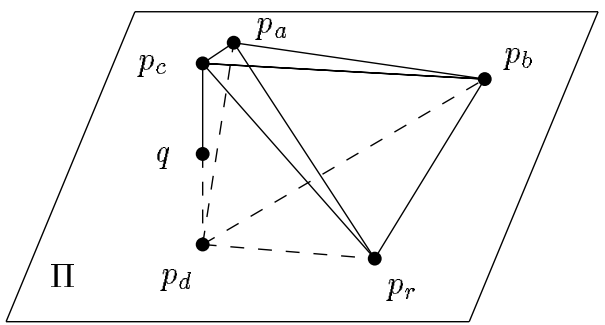

c)

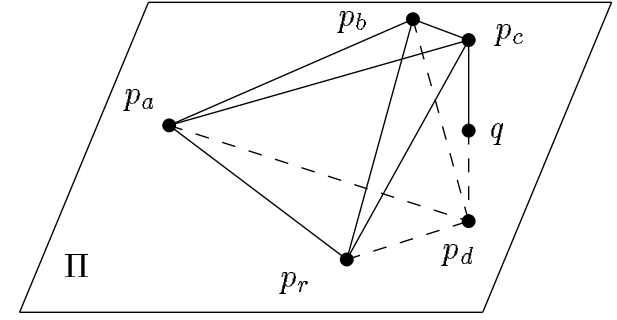

b)

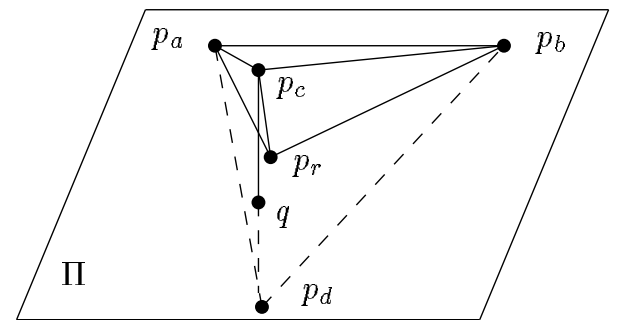

d)

Fig. 3: Four cases.

Case 2. Either $p_{i}=p_{c}$ or $p_{i}=p_{d}$. Suppose that $p_{i}=p_{c}$. By definition of $p_{i}$ the vertex $p_{b}$ has only three incident edges in $G_{Q},\left(p_{b} p_{a}\right),\left(p_{b} p_{c}\right)$, and $\left(p_{b} p_{d}\right)$. All of them are convex since $\left(p_{a} p_{b}\right)$ is convex. The points $p_{r}, p_{a}, p_{b}, p_{c}$ and $p_{d}$ form a circuit $\left(Z_{+}, Z_{-}\right)$where $Z_{+}=\left\{p_{r}, p_{b}\right\}$ and $Z_{-}=\left\{p_{a}, p_{c}, p_{d}\right\}$. The triangulation $T$ is supported on $\left(Z_{+}, Z_{-}\right)$and its flip makes the tetrahedron $\left(p_{a} p_{b} p_{c} p_{d}\right)$ which can be added to $T$. Contradiction.

(ii) It follows from Case 1.

By Lemma 8 the graph $G_{Q}^{\prime}$ is broken up into disjoint cycles. The following Lemma provides the final argument in the proof of Theorem 4.

Lemma 9 Let $T$ be a triangulation of $A$ in $\mathbb{R}^{3}$ and $\operatorname{rank}(T)<n-3$. There exists a vertex of $A \backslash H \cup\left\{p_{r}\right\}$ incident to at least three convex edges of $Q$.

Proof: The polytope $T_{\text {comp }}$ has a non-convex edge $e=\left(p_{a} p_{b}\right)$ by Lemma 5. The points $p_{a}$ and $p_{b}$ do not lie on the horizon $H$, otherwise $T$ is supported by either $e$ or its adjacent edge in $G_{Q}^{\prime}$. Let $R$ be the convex hull of the points on the horizon and $p_{r}$. The points $p_{a}, p_{b} \notin R$ because all the points are in convex position. Since the point $p_{a}$ is outside the convex polytope $R$, there is a face $f$ of $R$ such that the plane containing $f$ separates $R$ and $p_{a}$. Let $p_{j}$ be the vertex of $Q$ at largest distance from $p_{r}$ in the direction normal to $f$. 
It is impossible that all the edges of $Q$ incident to $p_{j}$ are non-convex. By Lemma 8 there are at least two convex edges of $Q$ incident to $p_{j}$. We show that there must be a third convex edge. Suppose to the contrary that $p_{j}$ has exactly two convex edges, say $p_{a} p_{j}$ and $p_{b} p_{j}$. Note that three points $p_{a}, p_{b}$ and $p_{j}$ are not collinear. Let $\Pi$ be the plane passing through $p_{a}, p_{b}$, and $p_{j}$. Suppose, for simplicity, that $\Pi$ is horizontal and $p_{r}$ is below $\Pi$. We project the edges of $Q$ incident to $p_{b}$ onto the plane $\Pi$ using the direction $p_{r} p_{j}$, see Fig 4. The edges $p_{a} p_{j}$ and $p_{b} p_{j}$ form two wedges in the projection plane, convex (with angle $<\pi$ ) and non-convex (with angle $>\pi$ ). Let $p_{j} p_{c}$ be any edge that is projected into the convex wedge $p_{a} p_{j} p_{b}$, see Fig 4 . The point $p_{c}$ lies on the same side of $\Pi$ as $p_{r}$ (below $\Pi$ ) since it is non-convex edge of $Q$.

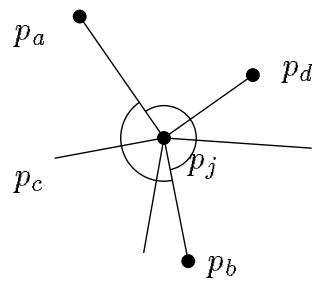

Fig. 4: Four cases. Note that there might be no edges projected into the convex wedge.

There must be at least one edge projected into the non-convex wedge $p_{a} p_{j} p_{b}$ (otherwise the face incident to both $p_{a} p_{j}$ and $p_{b} p_{j}$ has angle $>\pi$ ). Note that each such edges lies below $\Pi$ by the choice of $p_{j}$ (and there is no more points on $\Pi$ ). Among all such edges we choose the edge $p_{j} p_{d}$ making the smallest angle with $\Pi$. The edge $p_{j} p_{d}$ lies below or on $\Pi$ and is convex. Contradiction.

If the edge set of $G_{q}^{\prime}$ is not empty, then there is a vertex of degree at least three by Lemma 9. It contradicts Lemma 8 and, thus, Theorem 4 follows.

\section{Enumeration of triangulations}

We show that triangulations of a convex polytope can be reported efficiently. The algorithm is based on the reverse search technique of Avis and Fukuda [AF96]. The implementation is similar to the algorithm for enumerating planar triangulations [Bes00].

We define a triangulation tree $\mathcal{T}$ as follows. Let $A$ be a set of $n$ points in $\mathbb{R}^{3}$ in general and convex position and let $P$ be the convex hull of $A$. Let $\left(p_{1}, p_{2}, \ldots, p_{n}\right)$ be an arbitrary order of points in $A$. $\mathcal{T}$ is a spanning rooted tree of the graph of triangulations $G(A)$. The root $v_{\text {root }}$ of $\mathcal{T}$ corresponds to the unique triangulation $T_{\text {root }}$ of $P$ with rank $n-3$. Let $T$ be any triangulation different from $T_{\text {root }}$. There is an improving flip by Theorem 4 . Let $Z=\left(Z_{+}, Z-\right), Z_{+}=\left\{p_{a}, p_{b}\right\}, a<b, Z_{-}=\left\{p_{c}, p_{d}, p_{e}\right\}, c<d<e$ be a circuit such that $T$ is supported on $Z$. With the circuit $Z$ we associate the vector $v(Z) \in Z^{5}$ of indices $\{a, b, c, d, e\}$ sorted in increasing order. Abusing notation we call the vector of a circuit as the vector of corresponding flip $f$, i.e. $v(f)=v(Z)$. We define the parent flip $f_{\min }(T)$ which an improving flip with lexico-smallest vector $v\left(f_{\min }(T)\right)$. The node $v$ of $\mathcal{T}$ corresponding to $T$ is joined to the parent node corresponding to the triangulation obtained from $T$ by flipping $f_{\min }(T)$.

Let $f$ be a flip that can be applied to the current triangulation $T$ and let $Z$ be its circuit. Note that, for any vertex $p$ of $T$ participating in a flip $f$, the volume of the union of tetrahedra incident to $p$ changes when we apply the flip $f$, see Fig $1 \mathrm{~b}$. We call the flip $f$ positive if the volume of tetrahedra incident to the point of $Z$ with the smallest index (i.e. $p_{\min (a, b)}$ ) decreases when we apply the flip $f$ (note that positive $f$ can produce either two or three tetrahedra). Otherwise the flip $f$ is called negative. Let $F_{+}\left(F_{-}\right)$be the set of positive (negative) flips.

The enumerating algorithm basically performs the depth-first search on $\mathcal{T}$ with exception that the implementation is not recursive. For a current vertex of $\mathcal{T}$, we want to find efficiently its parent and sons. The parent flip can be obtained by finding the lexico-smallest flip in $F_{+}$. To characterize the son edges we introduce the following notation. 
Two circuits are neighbors if there is a tetrahedron whose vertices are contained in each circuit. A circuit can have $O(1)$ neighbors since a tetrahedron can participate in $O(1)$ circuits.

Let $Z$ be a circuit and $T^{\prime}$ be the triangulation obtained by the flip $f(Z)$ applied to the triangulation $T$. The circuit $Z$ is locally reversible if the flip $f(Z)$ is negative and each neighbor of $Z$ in the triangulation $T^{\prime}$ represents either a negative flip or a positive flip whose vector is lexico-smaller than vector $v(f(Z))$.

Lemma 10 (Son Edge) Let $T$ be a triangulation corresponding to a node $u$ of $\mathcal{T}$. Let $f$ be a flip which is not a neighbor of $f_{\min }(T)$. $f$ produces the triangulation of a son of $u$ if and only if $f$ is locally reversible and $v(f)$ is lexico-smaller than $v\left(f_{\min }(T)\right)$.

Proof: [Only if]: Suppose that $f$ produces the triangulation $T^{\prime}$ of a son of $u$. Let $Z$ be the circuit of $f$. The circuit $Z$ defines a positive flip of $T^{\prime}$ which is dual to $f$ and has the lexico-smallest vector among all positive flips of $T^{\prime}$. Then $f$ is locally-reversible flip of $T$. The vector of $f$ is lexico-larger than $v\left(f_{\min }(T)\right)$ since $f_{\min }(T)$ is still positive flip of $T^{\prime}$.

[If]: Let $f^{\prime}$ be the dual flip of $f$. Let $f^{\prime \prime}$ be a positive flip of $T^{\prime}$. If $f^{\prime \prime}$ is a neighbor of $f^{\prime}$, then $v\left(f^{\prime}\right)$ is lexico-smaller than $v\left(f^{\prime \prime}\right)$ because $f^{\prime}$ is locally reversible. If $f^{\prime \prime}$ is not a neighbor of $f^{\prime}$, then $f^{\prime \prime}$ is positive flip of $T$ and its vector is lexico-larger than $v\left(f_{\min }(T)\right.$.

The algorithm stores the current triangulation $T$ as the list $L_{v}$ of vertices, the list $L_{e}$ of edges, the list $L_{f}$ of faces, and the list $L_{t}$ of tetrahedra. With each tetrahedron $t \in L_{t}$, we store the pointers to its four faces. With each face $f \in L_{f}$, we store three pointers to its edges, two pointers to tetrahedra sharing $f$. With each edge of $L_{e}$, we store two pointers to its endpoints. The pointers allow to obtain, for example, vertices and edges of a tetrahedron in $\mathcal{T}$ in $O(1)$ time. We also store the list $L_{c}$ of all circuits of the triangulation $T$. Note that every tetrahedron can participate in at most four circuits which can be obtained using information stored at the list $L_{f}$.

The algorithm maintains the rank of current triangulation and the set of convex vertices of the polytope $Q$. The data structure contains the following trees.

- $T_{t}$, binary search tree of all tetrahedra in $T$

- $T_{+}$, binary search tree of all positive flips in $T$

- $T_{-}$, binary search tree of all negative flips in $T$

- $T_{r}$, binary search tree of all reversible flips $T$

The trees $T_{t}, T_{+}, T_{-}$, and $T_{r}$ store tetrahedra in lexicographical order of their vectors where the vector $v(\Delta) \in Z^{4}$ of a tetrahedron $\Delta$ is the sequence of indices of its vertices sorted in increasing order. We can combine two trees $T_{+}$and $T_{-}$since they store all flips (we use different trees to make a simple reduction to van Emde Boas data structure). The rank of current triangulation $T$ can be obtained by finding a tetrahedron $\Delta$ with the lexico-smallest vector in $T_{+}$by Theorem 4 . The rank of $T$ is the smallest index of vertices of $\Delta$ if $\Delta$ exists; otherwise the rank is $n-3$.

The algorithm can find flips corresponding to sons of a current node of the triangulation tree as follows. The flip $f_{\min }(T)$ can be found in $O(\log n)$ time using the tree $T+$. There is $O(1)$ neighbors of $f_{\min }(T)$. For each neighbor $f$ of $f_{\min }(T)$, the algorithm detects if $f$ is negative. For a negative flip $f$, the algorithm 1) applies the flip $f, 2$ ) maintains the trees $T_{t}, T_{+}, T_{-}$, and $T_{r}$ and 3 ) finds the parent flip for the resulting triangulation $T^{\prime}$, and 4) proceeds to the triangulation $T^{\prime}$ if $f_{\min }\left(T^{\prime}\right)$ is the dual flip of $f$. 
To process flips that are not neighbors of $f_{\min }(T)$ the algorithm scans locally reversible flips $f$ whose vectors are lexico-smaller than $v\left(f_{\min }(T)\right)$. The scanning procedure can be organized in such a way that next son flip can be found in $O(\log n)$ time. Using the data structure by van Emde Boas [vE77] for the trees $T_{t}, T_{+}, T_{-}$, and $T_{r}$ we can reduce the time to $O(\log \log n)$.

Theorem 11 Triangulations of a convex polytope in $\mathbb{R}^{3}$ can be reported in $O(\log \log n)$ time per triangulation.

\section{Conclusion}

We study the graph of triangulations whose vertices represent the triangulations and whose edges represent geometric bistellar flips. We proved that the graph of triangulations of a convex polytope in three dimensions is connected. We introduce a tree of triangulations and present an algorithm for enumerating triangulations in $O(\log \log n)$ time per triangulation. It would be interesting to clarify situation in higher dimensions $d \geq 4$. In what dimensions the graph of triangulations of a convex polytope is connected? Is there a way of efficient enumeration of triangulations using flips in dimension $d \geq 4$ ?

\section{References}

[AF96] D. Avis and K. Fukuda. Reverse search for enumeration. Discrete Appl. Math., 65:21-46, 1996.

[BE92] M. Bern and D. Eppstein. Mesh generation and optimal triangulation. In D.-Z. Du and F. K. Hwang, editors, Computing in Euclidean Geometry, volume 1 of Lecture Notes Series on Computing, pages 23-90. World Scientific, Singapore, 1992.

[Bes00] S. Bespamyatnikh. An efficient algorithm for enumeration of triangulations. Presented at 10th Annual Fall Workshop on Computational Geometry, 2000.

[Bjö95] A. Björner. Toplogical methods in combinatorics. In R. L. Graham, M. Grötschel, and L. Lovász, editors, Handbook of Combinatorics, pages 1819-1872. Elsevier, Amsterdam, 1995.

[BVS $\left.{ }^{+} 92\right]$ A. Björner, M. Las Vergnas, B. Sturmfels, N. White, and G. Ziegler. Oriented matroids. Cambridge University Press, Cambridge, 1992.

[dLSU99] J. A. de Loera, F. Santos, and J. Urrutia. The number of geometric bistellar neighbors of a triangulation. Discrete Comput. Geom., 21(1):131-142, 1999.

[EM90] H. Edelsbrunner and E. P. Mücke. Simulation of simplicity: A technique to cope with degenerate cases in geometric algorithms. ACM Trans. Graph., 9(1):66-104, 1990.

[ER98] P. H. Edelman and V. Reiner. Visibility complexes and the Baues problem for triangulations in the plane. Discrete Comput. Geom., 20(1):35-59, 1998.

[GKZ94] I. M. Gel'fand, M. M. Kapranov, and A. V. Zelevinsky. Discriminants, Resultants and Multidimensional Determinants. Birkhäuser, Boston, 1994. 
[HN99] F. Hurtado and M. Noy. Graph of triangulations of a convex polygon and tree of triangulations. Comput. Geom. Theory Appl., 13:179-188, 1999.

[Joe89] B. Joe. Three-dimensional triangulations from local transformations. SIAM J. Sci. Stat. Comput., 10:718-741, 1989.

[Law77] C. L. Lawson. Software for $C^{1}$-interpolation. In John Rice, editor, Mathematical Software III. Academic Press, New York, 1977.

[RZ96] J. Rambau and G. M. Ziegler. Projections of polytopes and the Generalized Baues Conjecture. Discrete Comput. Geom., 16(3):215-237, 1996.

[San00] F. Santos. Triangulations with very few geometric bistellar neighbors. Discrete Comput. Geom., 23(1):15-33, 2000.

[vE77] P. van Emde Boas. Preserving order in a forest in less than logarithmic time and linear space. Inform. Process. Lett., 6:80-82, 1977.

[Zie94] G. Ziegler. Lectures on Polytopes. Springer-Verlag, New York, 1994. 

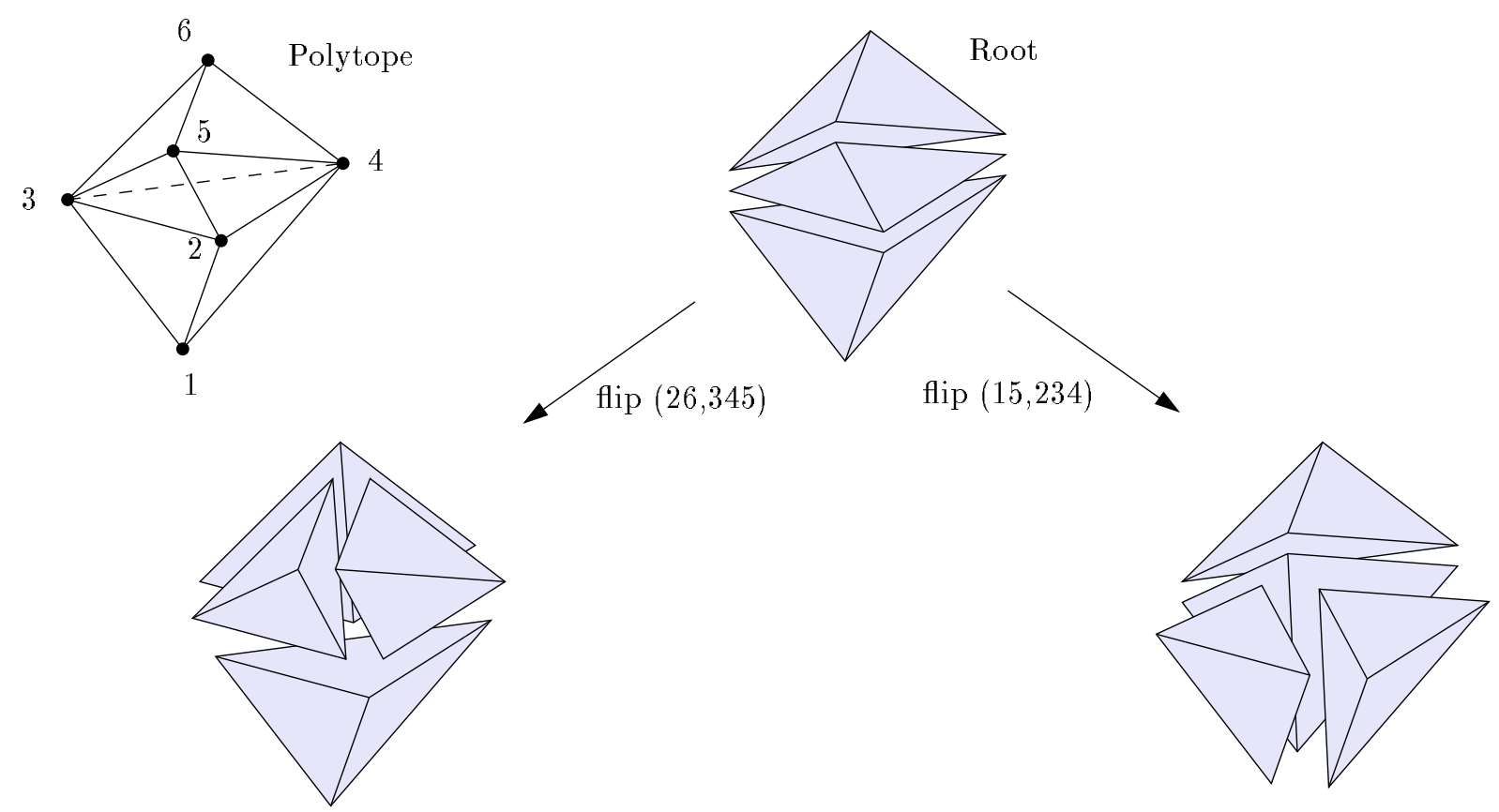

flip $(16,234)$
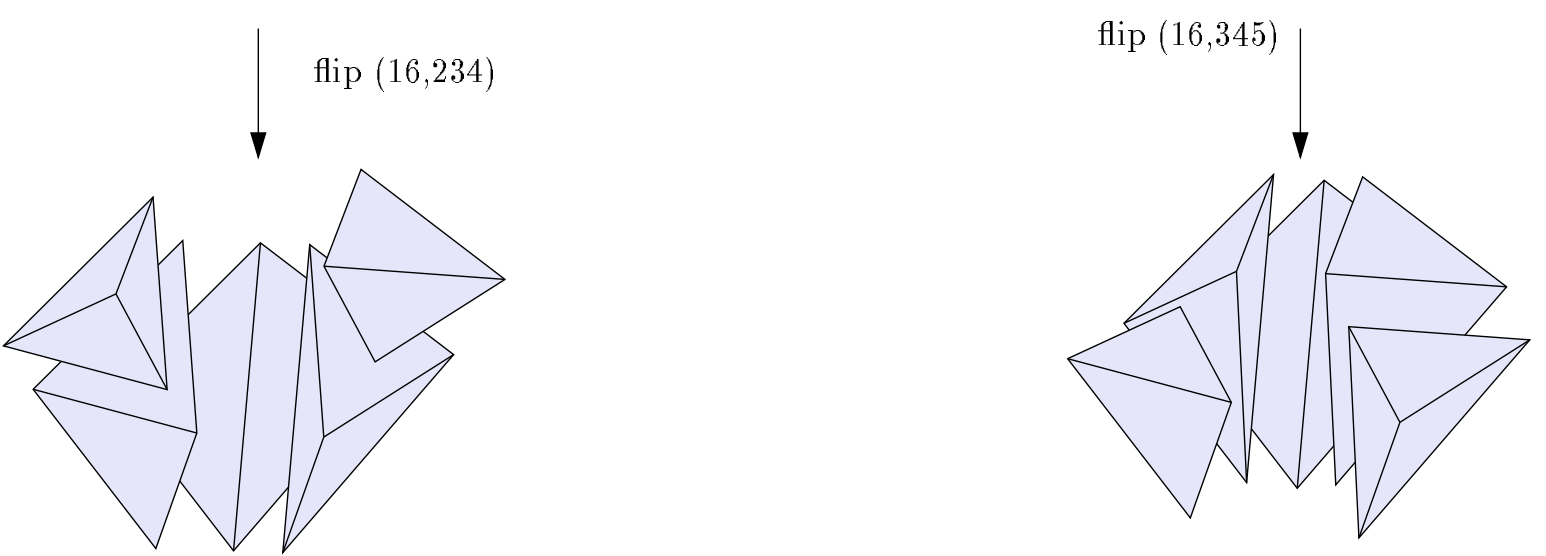

flip $(26,145)$

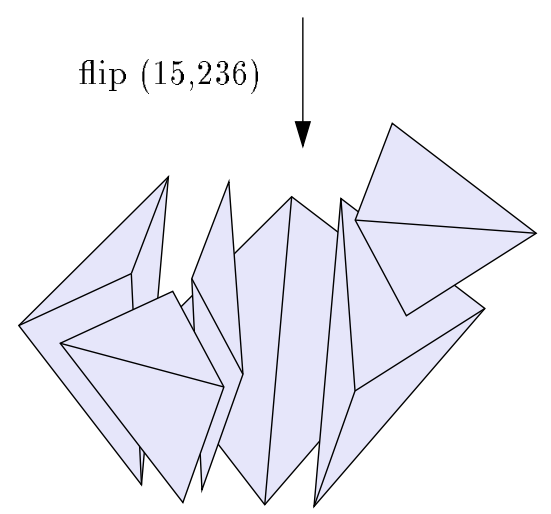

Fig. 5: Tree of triangulations. 